\title{
Towards a density of states approach for dense matter systems
}

\section{Kurt Langfeld ${ }^{* \dagger}$}

Research Centre for Mathematical Sciences, Plymouth University, Plymouth PL4 9DE, UK,

E-mail: kurt. langfeldeplymouth.ac.uk

\section{Jan Pawlowski}

Institute for Theoretical Physics, University of Heidelberg, 69120 Heidelberg, Germany

\section{Biagio Lucini}

Department of Physics, Swansea University, Swansea SA2 8PP, UK

\section{Antonio Rago}

Research Centre for Mathematical Sciences, Plymouth University, Plymouth PL4 9DE, UK

\section{Roberto Pellegrini}

Dipartimento di Fisica dell'Università di Torino, 10125 Torino, Italy

\begin{abstract}
The modified density-of-states method (LLR algorithm [1]) features an exponential error suppression and is not restricted to theories with positive probabilistic weight. It is applied to the $\mathrm{SU}(2)$ gauge theory at finite densities of heavy quarks. The key ingredient here is the Polyakov line probability distribution, which is obtained of over 80 orders of magnitude. We briefly address whether the exponential error suppression could be sufficient to simulate theories with a strong sign problem.
\end{abstract}

31st International Symposium on Lattice Field Theory LATTICE 2013

July 29 - August 3, 2013

Mainz, Germany

\footnotetext{
* Speaker.

${ }^{\dagger}$ This work is supported by STFC under the DiRAC framework. We are grateful for the support from the HPCC Plymouth.
} 


\section{Introduction:}

First principles simulations of QCD at finite baryon densities are an outstanding problem in particle physics due to the notorious sign problem. Insights into the QCD phase diagram (as a function of the chemical potential and the temperature) might be gained by considering the heavy quark limit: for $S U\left(N_{c}>2\right)$, those theories are still hampered by a sign problem, but there are indications [2] that the sign problem is less severe and solvable with recent techniques such as worm type algorithms [3, 4], complex Langevin techniques [5, 6] or the fermion bag approach [7]. Monte-Carlo simulations with respect to the "density of states" [8] rather than the (potentially complex) Gibbs factor might also be an interesting alternative to action based simulations $[1,9]$.

The starting point is the continuum formulation of $S U\left(N_{c}\right)$ Yang-Mills theories with the chemical potential $\mu$ for the heavy quarks with mass $m$. Using the heat-kernel approach, a systematic expansion of the quark determinant in powers of $1 / m$ yields the effective gluonic action (formulated in terms of links $U_{\mu}$ ) [10]:

$$
S[U]=S_{\mathrm{YM}}[U]+f p[U], \quad p[u]:=\sum_{\vec{x}} P(\vec{x})
$$

where $P(\vec{x})$, the (traced) Polyakov line, and $f$ is given by:

$$
P(\vec{x})=\frac{1}{N_{c}} \operatorname{tr} \prod_{t=1}^{N_{t}} U_{4}(\vec{x}, t), \quad f=\sqrt{2} \pi^{-3 / 2}(m T)^{3 / 2} a^{3} \exp \{(\mu-m) / T\},
$$

and where $N_{t}$ is the number of links in temporal direction, $a$ is the lattice spacing, $T=1 / N_{t} a$ the temperature and $S_{\mathrm{YM}}[U]$ is the (Wilson) action of pure Yang-Mills theory. Note that the only dependence on the spatial links is in the Wilson action of pure Yang-Mills theory. If we integrate over these links in leading order strong coupling expansion, we obtain an effective theory that only depends on the Polyakov lines and that features a nearest neighbour Polyakov line interaction. The effective theory is the so-called $S U\left(N_{c}\right)$ spin model [11, 4]. We here refrain from the strong coupling expansion, but will consider the theory with action (1.1), which can be considered the weak coupling version of the $S U\left(N_{c}\right)$ spin model. For $N_{c}=3$, this theory describes full QCD with heavy quarks at finite densities.

Given the close relation between the Polyakov line and colour confinement, a quantity of particular interest is the the Coleman effective potential for the Polyakov line. This potential is defined as usual by means of the Legendre transformation of the generating functional:

$$
\begin{aligned}
V(q) & =\frac{T}{V_{3}}(\mu q+j q-\ln Z[J]), \quad q=\frac{d \ln Z[j]}{d j}=\langle p[U]\rangle . \\
Z[j] & =\int \mathscr{D} U_{\mu} \exp \left\{S_{\mathrm{YM}}[U]+j \sum_{\vec{x}} P(\vec{x})\right\} .
\end{aligned}
$$

The generating functional $Z[j]$ would be obtained quite easily from the probability density $\rho(q)$ for finding a particular value for the (integrated) Polyakov line $q$ :

$$
\rho(q)=\int \mathscr{D} U_{\mu} \exp \left\{S_{\mathrm{YM}}[U]\right\} \delta\left(q-\sum_{\vec{x}} P(\vec{x})\right) .
$$


We find at least formally:

$$
Z[j]=\int d q \rho(q) \exp \{j q\} .
$$

In practice, it is challenging to obtain a statistically viable result for the effective potential $V(q)$ (1.3). There are several reasons for that: (i) Obtaining $\rho(q)$ by a standard histogram method is extremely costly since the standard deviation of the distribution decreases with the volume. For any value $q$ significantly different from zero, a large number of independent configurations is necessary, and one such configuration only produces one entry in the histogram. (ii) The $j q$ term in the Coleman potential (1.3) cancels to a great deal the $j \sum_{\vec{x}} P(\vec{x})$ term in the generating functional (1.4) as can be e.g. seen from a classical approximation. Those terms are extensive quantities and are potentially large. This leaves us with a poor signal-to-noise ratio. (iii) For $S U\left(N_{c}\right)>2, P(\vec{x})$ is complex, and standard Monte-Carlo method are no longer viable because of a sign problem. In the following, we will confine us to the $S U(2)$ case for which the sign problem is absent. We will resolve the issues (i) and (ii) by adopting a density-of-states approach that features an exponential error suppression.

\section{The density-of-states approach (LLR method)}

Let us consider the partition function $Z$ of a theory of the variable $\phi$ and action $S[\phi]$ :

$$
Z=\int \mathscr{D} \phi \exp \{\beta S[\phi]\}
$$

where is a solid-state physics context $\beta$ is the inverse temperature while in quantum field theory $\beta$ is interpreted as the inverse coupling strength. This partition function can be trivially rewritten using the so-called density-of-states $\rho$ :

$$
\begin{aligned}
\rho(E) & =\int \mathscr{D} \phi \delta(E-S[\phi]), \\
Z & =\int d E \rho(E) \mathrm{e}^{\beta E} .
\end{aligned}
$$

Knowledge of $\rho(E)$ reduces the calculation of the partition function to an ordinary integral. The partition function is at the heart of many interesting quantities such as the thermal energy density, pressure, latent heat (for theories with a 1st order phase transition) or interface tensions. However, the integral (2.3) is a real challenge: $\beta$ is usually of order 1 while the action $E$ is proportional to the lattice volume, which easily reaches a 100,000 for modest lattice sizes. It is only the lack of states at high actions that renders the integral (2.3) finite. How do we calculate then $\rho(E)$ with a sufficient precision to obtain significant values for $Z$ ?

Figure 1 shows an actual result for the density-of-states $\rho$ for the cases of a $\mathrm{SU}(2)$ and $\mathrm{SU}(3)$ gauge theory. It appears that $\log _{10} \rho(E)$ is a remarkable smooth function over the whole action range $\left[0, E_{\max }\right]$ with $E_{\max }=60,000$ for the present case. This motivates a piecewise linear Ansatz for this quantity:

$$
\rho_{L L R}(E)=\rho\left(E_{0}\right) \exp \left\{a\left(E_{0}\right)\left(E-E_{0}\right)\right\}, \quad E_{0}<E<E_{0}+\delta E .
$$



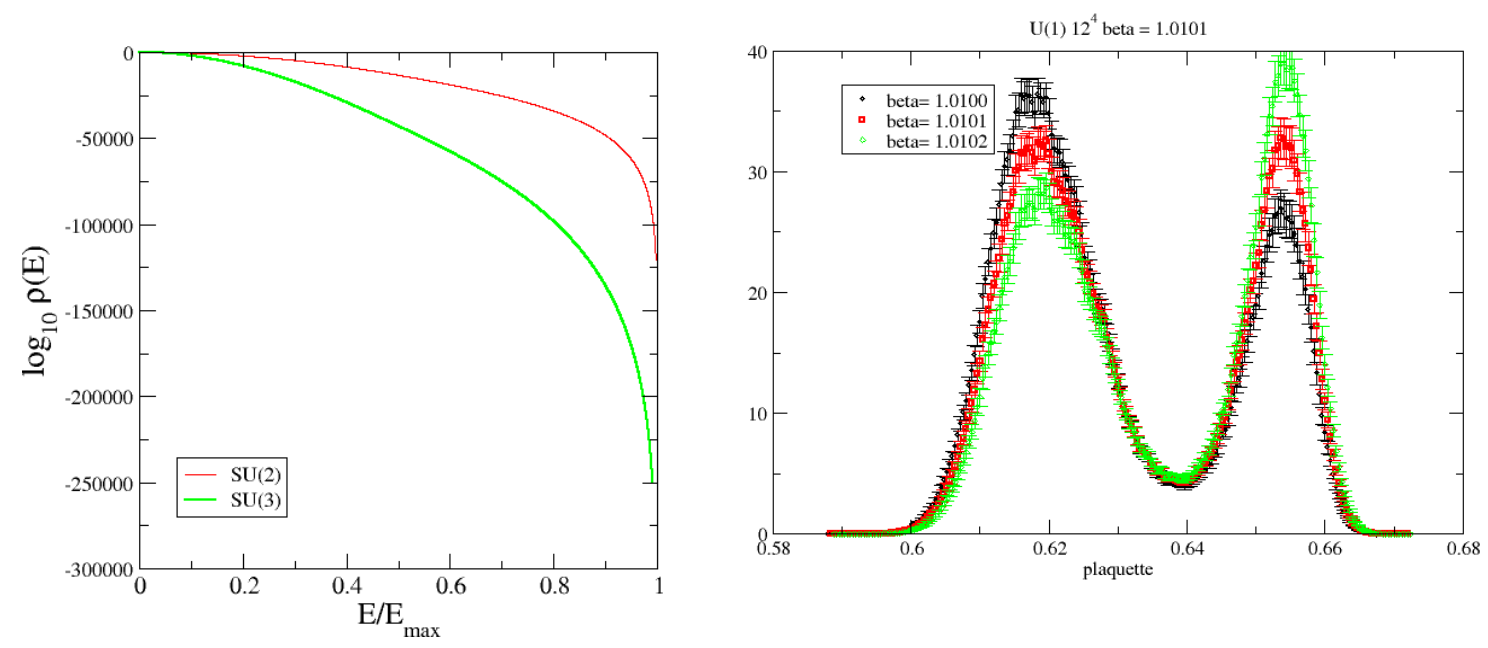

Figure 1: Left: The logarithm (base 10) of the density-of-states for a SU(2) and SU(3) lattice Yang-Mills theory for a $10^{4}$ lattice. Right: Probability distribution of the action for the compact U(1) theory for a $12^{4}$ lattice.

The task is now to find the expansion coefficients $a\left(E_{0}\right)$, which can be interpreted as derivatives to the partition function:

$$
a\left(E_{0}\right)=\left.\frac{1}{Z} \frac{d Z}{d E}\right|_{E=E_{0}} .
$$

Key ingredient to obtain $a\left(E_{0}\right)$ are truncated and re-weighted expectation values [1]:

$$
\begin{aligned}
\langle\langle f(S[\phi])\rangle\rangle(a) & =\frac{1}{\mathscr{N}} \int \mathscr{D} \phi f(S) \theta_{\left[E_{0}, \delta E\right]} \mathrm{e}^{-a S[\phi]}, \\
\theta_{\left[E_{0}, \delta E\right]} & =\left\{\begin{array}{l}
1 \text { for } E_{0} \leq S[\phi] \leq E_{0}+\delta E, \\
0 \text { else, }
\end{array}\right.
\end{aligned}
$$

The double-bracket expectation values can be evaluated using standard Monte-Carlo techniques: configurations are generated with respect to the Gibbs factor $\exp \{-a S[\phi]\}$ and rejected if the target configuration would produce an action that falls outside the allowed action window. Using the definition of the density-of-states $\rho(E)$ in (2.2), we can write:

$$
\langle\langle f(S)\rangle\rangle(a)=\frac{1}{\mathscr{N}} \int \mathrm{d} E f(E) \rho(E) \theta_{\left[E_{0}, \delta E\right]} \mathrm{e}^{-a E} .
$$

We now specialise to

$$
f(S[\phi])=S[\phi]-\left(E_{0}+\frac{\delta E}{2}\right)=: \Delta S[\phi] .
$$

If the parameter $a$ in (2.6) equals the exact value $a_{\mathrm{ex}}$, we obtain:

$$
\langle\langle\Delta S[\phi]\rangle\rangle\left(a_{\mathrm{ex}}\right)=\frac{1}{\mathscr{N}} \int \mathrm{d} E\left(E-E_{0}-\frac{\delta E}{2}\right) \theta_{\left[E_{0}, \delta E\right]}=0 .
$$

The latter equation can be solved for $a_{\mathrm{ex}}$. If $\langle\langle\Delta S[\phi]\rangle\rangle(a)$ is positive, the $a$ in the re-weighting factor $\mathrm{e}^{-a S[\phi]}$ is too small to compensate the exact density-of-states $\rho$. This suggests the fixed-point 

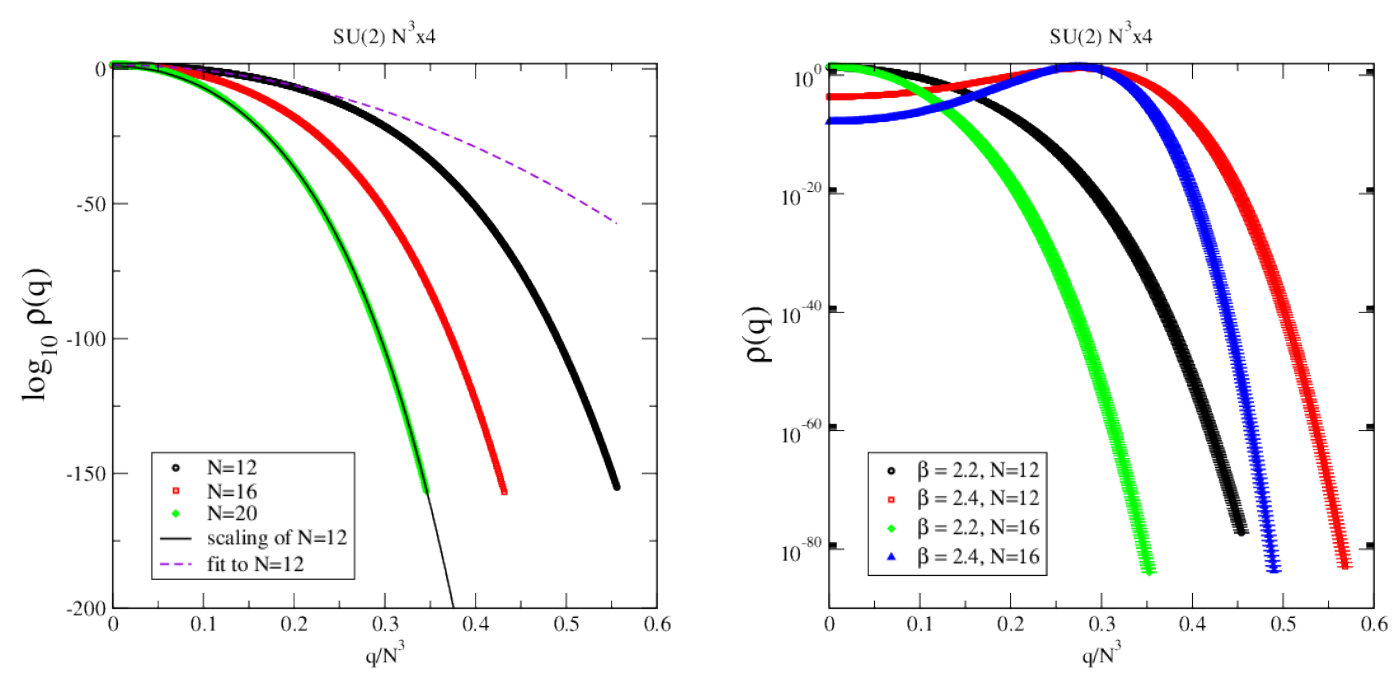

Figure 2: Left: The (integrated) Polyakov line probability distribution for $\mathrm{SU}(2)$ and a $N^{3} \times 4$ lattice. Right: Probability distribution for the confinement $(\beta=2.2)$ phase and for the deconfinement phase $(\beta=2.4)$.

iteration (with a suitable chosen relaxation parameter $\lambda>0$ ):

$$
a_{n+1}=a_{n}+\lambda\langle\langle\Delta S[\phi]\rangle\rangle\left(a_{n}\right), \quad a_{\mathrm{ex}}=\lim _{n \rightarrow \infty} a_{n} .
$$

Practical details on the implementation of the LLR algorithms will be presented in a forthcoming publication. The calculation of $a\left(E=E_{0}\right)$ is then carried out for a range of action values $E_{0}$ and the the density-of-states $\rho(E)$ is recovered from (2.4). The probability distribution for the action $E$ is then easily estimated from

$$
P(E ; \beta)=\rho(E) \exp \{\beta E\} .
$$

Error bars can be obtained by the standard bootstrap method. Given the set of $a$ values, this analysis takes little time on a standard desktop making it feasible to explore a quasi-continuum of $\beta$ values. This is particularly interesting to locate a 1st order phase transition. Figure 1, right panel, shows our findings for the compact $\mathrm{U}(1)$ gauge theory for a $12^{4}$ lattice.

The above approach to the action density-of-states can be easily generalised to calculate the probability distribution $\rho(q)$ (1.5) for the (integrated) Polyakov line $q$. To this aim, we set:

$$
\mathscr{D} \phi=\mathscr{D} U_{\mu} \exp \left\{S_{\mathrm{YM}}[U]\right\} .
$$

The LLR method now generates a quasi-continuum of probabilities $\rho_{\beta}(q) \exp \{j q\}$ as a function of the external source $j$. Note, however, that the generalised density-of-states $\rho_{\beta}(q)$ has to be re-calculated for each Wilson coefficient $\beta$.

\section{Numerical Results}

The first simulations targeted the Polyakov line probability distributions $\rho_{\beta}(q)$ for a $N^{3} \times 4$ lattice for the confinement phase $(\beta=2.2)$. We have studied this distribution for several lattice 

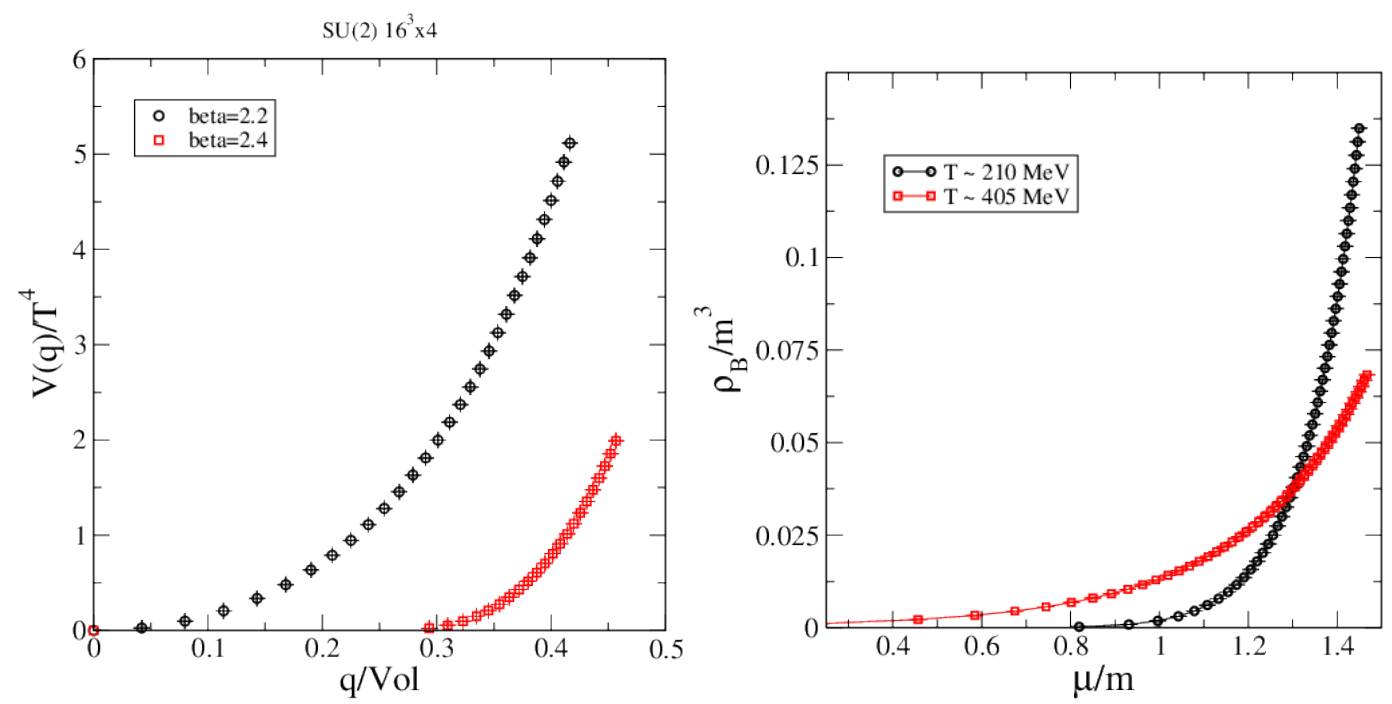

Figure 3: Left: The Coleman effective potential in units of the temperature $T$ for the $\mathrm{SU}(2)$ gauge theory for a $16^{3} \times 4$ lattice for the confinement $(\beta=2.2)$ and the high temperature $(\beta=2.4)$ phase. Right: For $\mathrm{SU}(2)$, the "baryon" (diquark) density as a function of the chemical potential $\mu$ in units of the heavy quark mass chosen to match the charm quark in QCD.

sizes [12]. With the LLR algorithm, we quite easily obtain the distribution over hundreds of orders of magnitude (see figure 2). Increasing the spatial value, decreases the width of the distribution. Also shown is a Gaussian fit to the data for $N=12$ (see dashed line in figure 2, left panel). We observe significant deviations from a Gaussian behaviour at large values $q$. We expect that $\log \rho(q)$ scales with the volume. To perform this consistency check using our numerical findings, we rescaled the data for $N=12$ to match with the data for $N=20$. The re-scaled data (line in figure 2) nicely fall on top of the data for $N=20$ (green symbols).

We then studied the probability distribution in the deconfinement phase by using $\beta=2.4$. The result is shown in figure 2, right panel. Due to spontaneous symmetry breaking, the distribution is suppressed for the "false vacuum" $q=0$. The suppression of $\rho(q=0)$ increases with increasing spatial volume resulting in a spontaneous breakdown of the centre symmetry in the infinite volume limit.

Our numerical results benefit from the exponential error suppression of the LLR algorithm making it possible to calculate the Coleman effective potential (1.3) by a direct Legendre transformation, Figure 3, right panel, shows our findings for a $16^{3} \times 4$ lattice. In the confinement phase ( $\beta=2.2$ ) the data points almost quadratically raise with increasing $q$ while in the high temperature phase ( $\beta=2.4$ ) the potential is strongly suppressed for small $q$ (in fact, vanishing in the infinite volume limit due to spontaneous symmetry breaking).

Finally, we show the "baryon", i.e., the diquark, density $\rho$ as a function of the chemical potential $\mu$ using the approximations leading to $(1.1,1.2)$. We have chosen "physical" parameters such as a string tension of $440 \mathrm{MeV}$ and a heavy quark mass matching that of the charm quark in QCD. In the confinement phase, we observe a sharp rise of the density when the quark chemical potential starts exceeding the quark mass gap. In the high temperature phase, we observe quite a 
significant density below the threshold. These contributions arise from temperature excitations of single quarks over the mass gap. This needs to be contrasted with the confinement phase where a diquark needs to be excited to obtain a contribution to the baryon density.

\section{References}

[1] K. Langfeld, B. Lucini and A. Rago, Phys. Rev. Lett. 109 (2012) 111601 [arXiv:1204.3243 [hep-lat]].

[2] Y. D. Mercado, H. G. Evertz and C. Gattringer, Phys. Rev. Lett. 106 (2011) 222001 [arXiv:1102.3096 [hep-lat]].

[3] N. Prokof'ev and B. Svistunov, Phys. Rev. Lett. 87 (2001) 160601.

[4] Y. D. Mercado and C. Gattringer, Nucl. Phys. B 862 (2012) 737 [arXiv:1204.6074 [hep-lat]].

[5] G. Aarts and F. A. James, JHEP 1201 (2012) 118 [arXiv:1112.4655 [hep-lat]].

[6] G. Aarts, F. A. James, J. M. Pawlowski, E. Seiler, D. Sexty and I. -O. Stamatescu, JHEP 1303 (2013) 073 [arXiv:1212.5231 [hep-lat]].

[7] S. Chandrasekharan and A. Li, JHEP 1101 (2011) 018 [arXiv:1008.5146 [hep-lat]].

[8] Fugao Wang and D. P. Landau, Phys. Rev. Lett. 86 (2001) 2050.

[9] A. Bazavov, B. A. Berg, D. Du and Y. Meurice, Phys. Rev. D 85 (2012) 056010 [arXiv:1202.2109 [hep-lat]].

[10] K. Langfeld and G. Shin, Nucl. Phys. B 572 (2000) 266 [hep-lat/9907006].

[11] F. Karsch and H. W. Wyld, Phys. Rev. Lett. 55 (1985) 2242.

[12] K. Langfeld and J. M. Pawlowski, arXiv:1307.0455 [hep-lat]. 